\title{
The Monte Carlo Program KORALZ, Version 4.0, for the Lepton or Quark Pair Production at LEP/SLC Energies
}

\author{
S. Jadach \\ CERN, Theory Division, CH 1211, Geneva 23, Switzerland, \\ and \\ Institute of Nuclear Physics, Kraków, ul. Kawiory 26a, Poland \\ B.F.L. Ward \\ Department of Physics and Astronomy, \\ The University of Tennessee, Knoxville, Tennessee 37996-1200, USA \\ and \\ SLAC, Stanford University, Stanford, California 94309, USA \\ and \\ Z. Wa̧s \\ CERN, Theory Division, CH 1211, Geneva 2马, Switzerland, \\ and \\ Institute of Nuclear Physics, Kraków, ul. Kawiory D6a, Poland
}

\begin{abstract}
The Monte Carlo program for $\tau$ pair production and decay applicable to the experiments in $e^{+} e^{-}$colliders in the $Z$ resonance energy range is presented. The program includes initial and final state bremsstrahlung corrections up to the $\mathrm{O}\left(\alpha^{2}\right)$ (with exclusive exponentiation), and $\mathrm{O}(\alpha)$ electroweak corrections. More than twenty distinct $\tau$ decay modes can be generated: (a) leptonic modes $\tau^{-} \rightarrow \bar{\nu} \nu e^{-}(\gamma)$ and $\bar{\nu} \nu \mu^{-}(\gamma)$; (b) one-meson modes, $\nu \pi^{-}$ and $\nu K^{-}$; (c) two-meson modes, $\nu\left\{\pi^{-} \pi^{0}\right\}_{\rho^{-}}, \nu\left\{K^{-} \pi^{0}\right\}_{K^{*-}}$ and $\nu\left\{K^{0} \pi^{-}\right\}_{K^{*-}}$; (d) threemeson modes, $\nu\left\{\pi^{-} 2 \pi^{0}\right\}_{a_{1}^{-}}, \nu\left\{2 \pi^{-} \pi^{+}\right\}_{a_{1}^{-}}, \nu K^{+} K^{-} \pi^{-}, \nu \bar{K}^{0} K^{0} \pi^{-}, \nu K^{-} K^{0} \pi^{0}, \nu K^{-} \pi^{0} \pi^{0}$, $\nu K^{-} \pi^{-} \pi^{+}, \nu K^{0} \pi^{0} \pi^{-}$and $\nu \eta \pi^{-} \pi^{0}$; (e) four-pion modes, $\nu \pi^{-} 3 \pi^{0}$, and $\nu 2 \pi^{-} \pi^{+} \pi^{0}$ (including the $\omega \pi^{-}$contribution); (f) multi-pion modes, $\nu n \pi^{ \pm, 0}$ with $n>4$; and finally the decay $\tau^{-} \rightarrow \nu \pi^{-} \pi^{0} \gamma$ from the $\omega \rightarrow \pi \gamma$. Complete $\mathrm{O}(\alpha)$ QED corrections are included in the leptonic decay channels, and for other decay channels an interface is provided to the PHOTOS Monte Carlo generator for approximate simulation of the QED corrections. The program includes three libraries of the pure electroweak corrections from the three independent groups of authors.

The program can be also used for generation of the final states different from $\tau^{+} \tau^{-}$ pairs, i.e., it is able to generate $\mu^{+} \mu^{-}, \nu \bar{\nu}$, and under some restrictions $u \bar{u}, d \bar{d}, c \bar{c}, s \bar{s}$ and $b \bar{b}$ final states.
\end{abstract}

To be submitted to Computer Physics Communications

CERN-TH 7075/93

November 1993 


\section{UPDATE SUMMARY}

Title of the program: KORALZ, version 4.0 .

Reference to original program: Comp. Phys. Commun. 66 (1991) 276.

Authors of original program: S. Jadach, B.F.L. Ward, and Z. Wạs

Computer: HP-9000/715, IBM 3090; Installation: CERN

Operating system: UNIX, VM/CMS

Programming language used: FORTRAN 77

High speed storage required: $<1 \mathrm{MB}$

No. of bits in a word: 32

Peripherals used: Line printer

No. of cards in combined program and test deck: about $19303+4961$ (or +4340 or +2259 depending on electroweak library chosen)

Keywords: Radiative corrections, heavy lepton $\tau$, Monte Carlo simulation, quantum electrodynamics, spin polarization, electro-weak theory.

Nature of the physical problem: Spin polarization of the $\tau$ in the process $e^{+} e^{-} \rightarrow$ $\tau^{+} \tau^{-}(n \gamma), \tau^{ \pm} \rightarrow X^{ \pm}$is used as an important data point for precise tests of the standard electroweak theory. The effects due to QED bremsstrahlung and apparatus efficiency have to be subtracted from the data. The program may be also applied for the production of $u, d, s, c, b$ quarks and neutrinos after appropriate redefinition of the coupling constants and masses. It may be used, as well, to simulate the muon pair production process. The precision of the program in the case of quarks is restricted.

Method of solution: The Monte Carlo simulation of the combined $\tau$ production and decay process is used to calculate the spin effects and effects of radiative corrections, including hard bremsstrahlung, simultaneously. Any experimental cut and apparatus efficiency may be introduced easily by rejecting some of the generated events.

Restrictions on the complexity of the problem: The incoming $e^{ \pm}$and outgoing $\tau^{ \pm}$may have only longitudinal polarizations. The total centre of mass energy is restricted to the range from about $20 \mathrm{GeV}$ to $150 \mathrm{GeV}$. High precision of the program is assured only in the region of the $Z$ where interference of bremsstrahlung from initial and final states can be neglected. When the program is used for the simulation of quark production, no hadronization, as well as no exclusive QCD corrections, are included.

Typical running time: Efficiency is 40 CPU sec. of HP-9000/715 per 1000 events, for the parameter setting as in the demonstration output. 


\section{Introduction}

The production of the $\tau$ lepton pair in the SLC/LEP experiment is recognised [1], [2] as a process which provides important experimental data for precise tests of the Standard Electroweak Theory [3].

The unique property of the process $e^{+} e^{-} \rightarrow \tau^{+} \tau^{-}$is that it is possible to measure spin polarization of the final state lepton $\tau$. This can be done by inspection of the energy distributions of $\tau$ decay products.

The unstable nature of the $\tau$, which on one hand constitutes its powerful virtue, on the other hand, complicates the data analysis. The presented Monte Carlo program is aimed as a helpful tool in the $\tau$ pair data analysis in SLC/LEP experiments.

In addition, after switching off $\tau$ decays, the program can be used to simulate the process of other fermion pair production in electron-positron annihilation $e^{+} e^{-} \rightarrow f \bar{f}(n \gamma)$, where the fermion may be a lepton, $f=\mu, \nu$, or under certain restrictions a quark, $q=u, d, s, c, b$.

The following important effects are taken into account:

1. Multiple QED hard bremsstrahlung from the initial and final state.

2. $O(\alpha)$ radiative corrections from the standard electroweak model [3].

3. Longitudinal spin polarization of the beams.

4. The following decay channels of the $\tau$ lepton $\tau^{-} \rightarrow \bar{\nu} \nu e^{-}(\gamma), \bar{\nu} \nu \mu^{-}(\gamma), \nu \pi^{-}, \nu K^{-}$, $\nu\left\{\pi^{-} \pi^{0}\right\}_{\rho^{-}}, \nu\left\{K^{-} \pi^{0}\right\}_{K^{*-}}, \nu\left\{K^{0} \pi^{-}\right\}_{K^{*-}}, \nu\left\{\pi^{-} 2 \pi^{0}\right\}_{a_{1}^{-}}, \nu\left\{2 \pi^{-} \pi^{+}\right\}_{a_{1}^{-}}, \nu K^{+} K^{-} \pi^{-}$, $\nu \bar{K}^{0} K^{0} \pi^{-}, \nu K^{-} \bar{K}^{0} \pi^{0}, \nu K^{-} \pi^{0} \pi^{0}, \nu K^{-} \pi^{-} \pi^{+}, \nu K^{0} \pi^{0} \pi^{-}, \nu \eta \pi^{-} \pi^{0}, \nu \pi^{-} 3 \pi^{0}, \nu 2 \pi^{-} \pi^{+} \pi^{0}$ (including the $\omega \pi^{-}$contribution), $\nu n \pi^{ \pm, 0}$ with $n>4$, and the decay $\tau^{-} \rightarrow \nu \pi^{-} \pi^{0} \gamma$ from the $\omega \rightarrow \pi \gamma$.

5. Spin polarization effects in the $\tau$ decay process (except of the multi-pion decays $\nu n \pi$, with $n>4$ ).

6. $\mathrm{O}(\alpha)$ bremsstrahlung in the leptonic $\tau$ decay modes: $\bar{\nu} \nu e^{ \pm}, \bar{\nu} \nu \mu^{ \pm}$and single (or double) bremsstrahlung in the leading logarithmic approximation for other decay channels.

The present version of KORALZ differs from the previous [4] version 3.8 in the following respect:

1. The top mass dependence of the electroweak corrections important in the heavy top mass regime is introduced.

2. Multiphoton final state radiation is introduced.

3. New $\tau$ decay modes and refinements on the existing ones are introduced. In particular, a matrix element algorithm for $\mathrm{O}(\alpha)$ radiative corrections in leptonic decays is introduced. 
4. Final states are coded in the PDG common block [5].

5. The meaning of the input parameter NPAR(4) is changed. The additional input parameter KEYYFS=NPAR (12) is introduced.

6. QED interference in the single bremsstrahlung mode of operation can be switched on and off with the help of input parameter NPAR(4).

The program has some limitations. Let us list them in the order of their importance:

1. The QED initial-final state bremsstrahlung interference in the presence of the multiple QED hard bremsstrahlung is not included.

2. The complete QED $O(\alpha)$ corrections to the $\tau$ decay are included only for the two $\bar{\nu} \nu e^{ \pm}, \bar{\nu} \nu \mu^{ \pm}$, leptonic decay channels.

3. There is no possibility to vary, from event to event, the flavour of the final state fermion and the energy of the beams.

4. The multi-pion $\tau$ decay modes (five $\pi$ 's and more) in $\tau$ decay do not include spin effects and are generated with an over-simplified matrix element.

5. The incoming $e^{ \pm}$and outgoing $\tau^{ \pm}$may have only longitudinal polarizations.

6. Precision of the program in the case of quarks as final states is restricted.

The KORALZ program has a modular structure. This separation assures clear structure of the program. It is especially important in the case of electroweak corrections. All of its basic parts, i.e., Monte Carlo photon emission generators YFS2/YFS3 [6, 7, 8], PHOTOS [9], MUSTRAAL [10], $\tau$ decay generator TAUOLA [11], and pure electroweak correction libraries DIZET [12], Z0POLE [13], were already published and are included in KORALZ either directly or with only minor changes. The status of the new multiphoton generator is such that the results from this program (cross sections and forward-backward asymmetries) were published in Ref. [7] and the program was distributed and used in all LEP collaborations. In particular, it was used in searches for abnormal emission of massive photon pairs in the $Z$ decays, see, for instance, paper of L3 collaboration [14]. The code of YFS3 program is available from the authors and will not change before publication [8]. For the completeness, we also include in KORALZ the third electroweak library of Hollik which corresponds to Ref. [15] and its code will be also updated and published in the future [16].

KORALZ includes spin polarization of the beams and the complete $\mathrm{O}(\alpha)$ virtual corrections from the (electroweak) standard model $[12,13,16]$, also hadronic and gluonic corrections. In the multi-bremsstrahlung mode, KORALZ uses a multi-photon algorithm for QED bremsstrahlung [6, 7, 8]. In the single bremsstrahlung mode, KORALZ emulates the program MUSTRAAL of Ref. [10]. In our case, however, the massive kinematics for the outgoing fermions is implemented in a better way than in the MUSTRAAL. 
QED corrections to the spin effects are implemented at the leading-log level only. With increasing precision of the experimental data, this approximation will require reexamination [17]. Users interested in more detailed information on the implementation of spin effects in KORALZ are referred also to paper [18]. Ref. [19] provides more information on the generation of the single final state bremsstrahlung and the way of combining the electroweak and final state bremsstrahlung corrections with the rest of QED corrections. This mode of operation is kept for the purpose of backward compatibility.

The $\tau$ decay library TAUOLA, used in KORALZ, is described in Ref. [11] and the corresponding leading-log radiative corrections in decays are generated as described in Ref. [9].

A few remarks on the limitations of the program: The total centre of mass energy is restricted to the range from about $20 \mathrm{GeV}$ to $150 \mathrm{GeV}$. One should keep in mind that the interference of bremsstrahlung from initial and final states can be neglected only close to the $Z$ resonance [20]. ${ }^{1}$ Let us remind the reader that the program KORALB of Ref. [22] for $\tau$ pair production at $\sqrt{s} \leq 30 \mathrm{GeV}$ includes all effects due to the finite mass of $\tau$ and due to transverse polarizations of $\tau^{ \pm}$and $e^{ \pm}$. KORALZ neglects the transverse spin correlations in $\tau$ decays. KORALB may serve for checks on the importance of these correlations [17]. KORALB includes $Z$ exchange at the Born level and single-bremsstrahlung only for $s$-chanel $\gamma$ exchange.

The outline of the paper is the following: In Chapter 2, we describe the functional structure of the program, in Chapter 3, it is explained how to use the program and in Chapter 4, the future developments of the program are discussed. In addition, Appendix A covers a detailed description of the interface between electroweak libraries and the rest of KORALZ and Appendix B explains modifications of the algorithm necessary in the quark and $\nu$ modes. In Appendix $\mathrm{C}$, it is explained how to reweigh already generated and stored events and, in this way, change effectively the value of some input parameters in the presence of the realistic experimental cuts. In Appendix D, we collect published important tests of the program and benchmarks. Finally, an extract from the output of the demonstration program is included at the end of the paper.

For the sake of completeness and convenience of the reader, we include here all information from our documentation on the previous version 3.8 of the program [4].

\section{The Structure of the Program}

The program consists of three distinct parts which are playing a different role and are, by the intention of the authors, well separated into corresponding modules with a well defined flow of information. They are as follows:

(1) The part of the program which simulates fermion pair production (no decay), including QED bremsstrahlung. In this part, the inclusion of all spin effects and electroweak corrections is administered. Two types of the QED bremsstrahlung

\footnotetext{
${ }^{1}$ For a precision better than $1 \%$, the size of the interference correction should be checked with the help of the single bremsstrahlung mode of KORALZ, as explained in Ref. [21].
} 
generators are employed: the YFS2/YFS3 generator of Refs. $[6,7,8]$ and a modified version of MUSTRAAL [10] of Ref. [18].

(2) The Monte Carlo library TAUOLA [11] of the programs for simulating practically all accessible experimentally exclusive decays of the polarised $\tau$ 's.

(3) Three libraries of the $\mathrm{O}(\alpha)$ electroweak radiative corrections in the standard electroweak theory according to Refs. [12,13] and Ref. [15].

The four momenta of final state fermions and of additional bremsstrahlung photons are generated in part (1). The basic generator in this part is the YFS2/YFS3 program of Ref. [6] which generates the fermion pair and multiple initial and/or final state photons. The algorithm of Ref. [19] is used subsequently in order to include the electro-weak corrections. The other, single bremsstrahlung generator, similar to that of MUSTRAAL, is also included; its main purpose are tests on the size of QED initial-final state interference. In the past, it was widely used as a basic program for fermion pair production at PETRA/PEP. A rather detailed description may be found in Ref. [10]. Three important improvements are made with respect to the original program: longitudinal spin polarization of $e^{ \pm}$and $\tau^{ \pm}$is introduced according to the prescription of Ref. [18]. The final state fermions may have a mass comparable to beam energy (improved kinematics, but not matrix element). For the $\gamma-Z$ boxes and soft bremsstrahlung interference exact matrix element was introduced. In the case of the multi-photon generator YFS2/YFS3, the initial-final state bremsstrahlung interference is neglected. This is to some extent justified in the region of the $Z$ peak [20] and in the absence of strong cut-offs. The production part of the program is supplemented with the subroutine TRALOR4 which performs the Lorentz transformation from the final fermion rest frame $\left(\tau^{ \pm}\right)$to the laboratory CMS frame. This subprogram is used in the TAUOLA library.

In the present version, the $\tau$ decay part (2) consists of the TAUOLA library which is able to simulate $\tau$ decays into the following channels: $\tau^{-} \rightarrow \bar{\nu} \nu e^{-}(\gamma), \bar{\nu} \nu \mu^{-}(\gamma), \nu \pi^{-}$, $\nu K^{-}, \nu\left\{\pi^{-} \pi^{0}\right\}_{\rho^{-}}, \nu\left\{K^{-} \pi^{0}\right\}_{K^{*-}}, \nu\left\{K^{0} \pi^{-}\right\}_{K^{*-}}, \nu\left\{\pi^{-} 2 \pi^{0}\right\}_{a_{1}^{-}}, \nu\left\{2 \pi^{-} \pi^{+}\right\}_{a_{1}^{-}}, \nu K^{+} K^{-} \pi^{-}$, $\nu \bar{K}^{0} K^{0} \pi^{-}, \nu K^{-} \bar{K}^{0} \pi^{0}, \nu K^{-} \pi^{0} \pi^{0}, \nu K^{-} \pi^{-} \pi^{+}, \nu K^{0} \pi^{0} \pi^{-}, \nu \eta \pi^{-} \pi^{0}, \nu \pi^{-} 3 \pi^{0}, \nu 2 \pi^{-} \pi^{+} \pi^{0}$ (including the $\omega \pi^{-}$contribution), $\nu n \pi^{ \pm, 0}$ with $n>4$ and the decay $\tau^{-} \rightarrow \nu \pi^{-} \pi^{0} \gamma$ from the $\omega \rightarrow \pi \gamma$. Complete $\mathrm{O}(\alpha)$ QED corrections are included in the leptonic decay channels, and for other decay channels an interface is provided to the PHOTOS Monte Carlo generator for approximate (leading logarithmic) simulation of the QED corrections. A more detailed description of TAUOLA and its interface to KORALZ is available separately [11].

The electroweak corrections in part (3) are described in Ref. [12], see also Ref. [23], and in Refs. $[13,15]$. These libraries of electroweak radiative corrections from the standard model include also the gluonic (hadronic) effects. The interface between electroweak libraries and KORALZ is described in Appendix A.

Let us now comment briefly on the interrelation among the above three parts of the program. The spin amplitudes from part (3) are used in part (1), in the final stage on the M.C. shaping of the $\tau^{+}, \tau^{-}$and $\gamma^{\prime}$ 's sample. The effects due to GSW corrections are added by a rejection technique. The programs in the decay part (2) use, in the simulation of 
the $\tau$ decay, the spin polarization vectors of $\tau^{+}$and $\tau^{-}$provided by the production part ${ }^{2}$ (1). In fact, most of the exchange of the information among the parts (1-3) goes through routine KORALZ which plays a role of the central manager subprogram. In particular, all initializations necessary to initiate the work of all the parts (1-3) are administrated by KORALZ. The calculation of $\sin ^{2} \theta_{W}$ (by electroweak library and using its $\sin ^{2} \theta_{W}$ definition) out of the muon lifetime, the $M_{Z}$ and top and Higgs masses is also administrated within KORALZ during the initialization mode.

\section{How to Use the Program}

A Monte Carlo event, i.e., a complete set of final state momenta and flavours, is generated by a single call to routine KORALZ. For example, a complete sequence of calls which generates 1000 M.C. events may look like

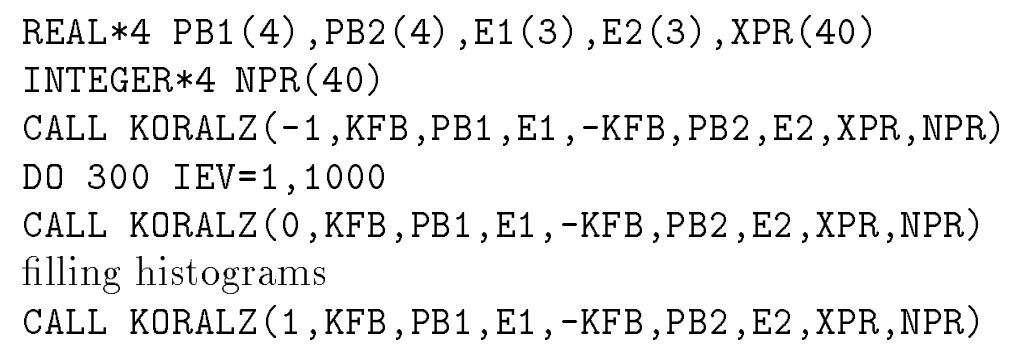

Here, the first call (obligatory) on KORALZ sets the $e^{ \pm}$beam momenta and transfers other input parameters (so far no event generation), the second call generates a M.C. event, and the third call (optional) terminates the generation by printing some useful output and calculating the total cross section, partial widths in $\tau$ decays, and so on, corresponding to a generated sample. Generally, the input parameters are transferred as KORALZ parameters and the output results (momenta, flavours) are encoded in certain common blocks, described later in this Section.

Let us explain now in more detail the parameters of KORALZ. As is obvious from the above example, the first parameter MODE in KORALZ (MODE , . . ) is simply saying whether KORALZ is called in the initialisation mode $(M O D E=-1)$, generation mode (MODE $=0$ ), or postgeneration mode (MODE=1).

\section{Initialisation Mode}

In the initialisation mode $(M O D E=-1)$ the meaning of the parameters in KORALZ (MODE, KFB , PB1 , E1 , -KFB , PB2 , E2 , XPR , NPR)

is the following: REAL*4 PB1 (4), PB2 (4) are four-momenta of the first and second beam (with PB1 (4) = PB2(4) being the energy). Both momenta are necessarily along a third

\footnotetext{
${ }^{2}$ Note the difference, with respect to the KORALB program, where the full spin correlations are taken into account. In KORALZ, only the helicities (in fact chiralities) of the $\tau^{ \pm}$are passed to the decay routines. For the hard bremsstrahlung configurations spin, quantization axes are also biased (at order of $\left.\mathrm{O}\left(m_{\tau}^{2} / s\right)\right)$. These uncertainties will be also studied in Ref. [17].
} 
axis in the laboratory system (CMS) and PB1 is required to have a positive third component. INTEGER*4 KFB is a flavour identifier of the first beam (i.e., associated with PB1). $\mathrm{KFB}=11,-11$ denotes electron and positron, respectively. Two four vectors REAL*4 E1(3), E2 (3) are spin polarization vectors of the first and second beam in the corresponding $e^{ \pm}$ rest frames. For the first beam (PB1,E1), the rest frame used to define its spin polarization vector $\mathrm{E} 1$ has its third axis directed along its line of flight, i.e., it is a standard helicity axis. For E2, the direction of the corresponding third axis is opposite to its line of flight, i.e., opposite to its helicity axis. For example, $\mathrm{KFB}=-11, \mathrm{E} 1=(0.0,0.0,1.0)$, $\mathrm{E} 2=(0.0,0.0,1.0)$, means that the user asks for the first beam being a right-handed positron and the second being a left-handed electron. Partial polarization is allowed, i.e., $\mathrm{E} 2=(0.0,0.0,0.5)$ would represent a $50 \%$ polarized second beam. However, no transverse beam polarization is allowed. The flavour of the first beam defines also the flavour of the first $\tau$. In the following, we will assume that $\mathrm{KFB}=-11$, and we will call the first $\tau$ the $\tau^{+}$. We recommend this setting because it was used in most of our tests. If the first beam was chosen to be $e^{-}(\mathrm{KFB}=11)$, the first $\tau$ will be automatically defined as $\tau^{-}$ and not as $\tau^{+}$. In this case, the user of KORALZ should keep in mind this redefinition when reading the rest of the paper. The other input parameters are transferred through matrices REAL*4 XPR (40) and INTEGER NPR (40).

In the table below, we give a short summary of all input parameters. For the masses energies and momenta, we use $G e V$, units if not stated otherwise. Note, that as a default input parameters (see output of demonstration program), we do not use "best experimental values." It is up to the user to make an up-to-date choice.

The important comment on the practical use of the electroweak libraries and the meaning of the KEYWLB are in order here. First let us remind the reader that due to notorious features of the FORTRAN77 it is not possible to isolate names of procedures and common blocks in various parts of the program. Electroweak libraries from three different group of authors $[12,13,15]$ have coincidental names. The "user friendly" solution would be to change them such that they differ. This costs, however, additional work, makes these programs incompatible with their published documentation and may introduce bugs. The above solution was, nevertheless, adopted in the previous version of the KORALZ at the time when codes of electroweak libraries were not yet stabilised and published. This solution, if the need arises, can be restored rather easily. Now, in the present version, we have adopted another solution: In the basic file of KORALZ the source code of the library is not included. In order to switch to one of them $[12,13,15]$ the user will have to load one of the additional files, including the particular electroweak library and interface. 


\begin{tabular}{|c|c|}
\hline Parameter & Meaning \\
\hline $\mathrm{KFB}$ & $\begin{array}{l}\text { Flavour identifier of the first beam, KFB }=11,-11 \text { denotes electron } \\
\text { and positron, respectively (PDG notation). }\end{array}$ \\
\hline PB1 & Four momentum of the first beam. PB1(3)>0 necessarily. \\
\hline E1 & Spin polarization vector of the first beam. E1(3)=twice helicity. \\
\hline PB2 & Four momentum of the second beam. \\
\hline E2 & $\begin{array}{l}\text { Spin polarization vector of the second beam. }-\mathrm{E} 2(3)=\text { twice he- } \\
\text { licity. }\end{array}$ \\
\hline $\operatorname{NPR}(1)=\operatorname{ISPIN}$ & $\begin{array}{l}\text { Switch for spin effects in } \tau \text { decay. Normally the user should set } \\
\text { ISPIN }=1 \text {, and when ISPIN }=0 \text { spin polarization effects in the de- } \\
\text { cays are absent. }\end{array}$ \\
\hline $\operatorname{NPR}(2)=$ INRAN & $\begin{array}{l}\text { Initialization constant, INRAN } \geq 1 \text {, for random number generator } \\
\text { used in the } \tau \text { pair production part of M.C., obsolete. }\end{array}$ \\
\hline $\operatorname{NPR}(3)=\mathrm{KEYGSW}$ & $\begin{array}{l}\text { Implementation level of the GSW standard electroweak model. } \\
\text { For KEYGSW=0, there is no } \gamma \text { exchange in the } s \text { channel at all } \\
\text { (only } Z \text { ). For KEYGSW }=1, \gamma \text { and } Z \text { exchange is included (also in } \\
\text { the presence of hard bremsstrahlung), but no vacuum polarization } \\
\text { is included. KEYGSW }=2,3 \text { are obsolete. For KEYGSW=4 QED } \\
\text { and electroweak corrections are combined together using a leading- } \\
\text { log inspired approach [19]. }\end{array}$ \\
\hline
\end{tabular}




\begin{tabular}{|c|c|}
\hline Parameter & Meaning \\
\hline $\operatorname{NPR}(4)=\mathrm{KEYRAD}$ & $\begin{array}{l}\text { Switch for the QED bremsstrahlung. Note, that some entries have } \\
\text { different meaning with respect to KORALZ version } 3.8 \text { ! } \\
\text { KEYRAD }=0 \text { means no QED bremsstrahlung. } \\
\text { KEYRAD }=1 \text { denotes single bremsstrahlung. } \\
\text { KEYRAD }=2 \text { denotes single bremsstrahlung, but QED interference } \\
\text { switched off. } \\
\text { KEYRAD=10 denotes single initial state bremsstrahlung with an "ad } \\
\text { hoc" exponentiated photon spectrum. } \\
\text { KEYRAD=12 default, option of KORALZ. Provides exponentiated } \\
\text { initial and/or final state bremsstrahlung according to the algorithm of } \\
{[6,7,8] \text {. See also NPR (12), new steering parameter of YFS2/YFS3. }} \\
\text { KEYRAD=11 as does KEYRAD=12, but with different kinematics } \\
\text { of electroweak corrections implementation and boosts from } \tau^{ \pm} \text {rest } \\
\text { frames to the laboratory system. } \\
\text { KEYRAD=111, } 112 \text { are included for backward compatibility with ver- } \\
\text { sion 3.8. Then only in the initial state, bremsstrahlung is exponenti- } \\
\text { ated and single final state bremsstrahlung is generated as in the default } \\
\text { option of KORALZ } 3.8 \text {. }\end{array}$ \\
\hline $\operatorname{NPR}(5)=\mathrm{JAK} 1$ & $\begin{array}{l}\text { The variable JAK } 1=1,2,3, \ldots \text { defines the decay channel of } \tau^{+} \text {as be- } \\
\text { ing } \bar{\nu} e^{+} \nu, \bar{\nu} \mu^{+} \nu, \bar{\nu} \pi^{+} \text {, and so on, accordingly to the order given in the } \\
\text { description of TAUOLA [11]. Furthermore, setting JAK1=0 causes } \\
\text { the } \tau^{+} \text {to decay into all channels according to predefined branching } \\
\text { ratios (see routine JAKER), and setting JAK1=-1 inhibits the decay } \\
\text { completely. }\end{array}$ \\
\hline $\operatorname{NPR}(6)=\mathrm{JAK} 2$ & The possible assignments of JAK2, for $\tau^{-}$, are the same as of JAK1. \\
\hline $\operatorname{NPR}(7)=\operatorname{ITFIN}$ & $\begin{array}{l}\text { This switch key allows to generate final state fermion pairs other } \\
\text { than } \tau \text { pairs. The } \tau \text { pairs are produced with ITFIN }=1, \mu \text { pairs with } \\
\text { ITFIN }=2, \nu \text { pairs with ITFIN }=3 \text {. The quarks are produced with } \\
\text { ITFIN }=501-506 \text {. Quark masses are taken directly from KORALZ in- } \\
\text { put. For ITFIN }>1 \text {, the } \tau \text { decay is automatically suppressed. }\end{array}$ \\
\hline $\operatorname{NPR}(8)=\operatorname{ITDKRC}$ & Switch for bremsstrahlung in the $\tau^{-}$decay. $1 / 0=$ on $/$ off. \\
\hline $\operatorname{NPR}(9)=\mathrm{KEYWLB}$ & $\begin{array}{l}\text { Type of the electroweak library in use. At present, only KEYWLIB=1 } \\
\text { is allowed. Choice of the library has to be performed at the loader/linker } \\
\text { level (without any modification in the source code). }\end{array}$ \\
\hline $\operatorname{NPR}(11)=\operatorname{NNEUT}$ & $\begin{array}{l}\text { Number of the neutrino species. Relevant only in the neutrino mode. } \\
\text { It is not passed to the electroweak libraries and thus, has no effect on } \\
\text { the total width of } Z \text {. }\end{array}$ \\
\hline
\end{tabular}




\begin{tabular}{|c|c|}
\hline Parameter & Meaning \\
\hline $\operatorname{NPR}(12)=\mathrm{KEYYFS}$ & $\begin{array}{l}\text { Steering parameter of the YFS2/3 program, assignments } 1000001 \text {, } \\
1000010 \text { and } 1000011 \text { denote, respectively, initial state brems- } \\
\text { strahlung, final state bremsstrahlung and both of them simul- } \\
\text { taneously. For other options, see Ref. [8] or comments in the } \\
\text { YFS3 source code. }\end{array}$ \\
\hline $\operatorname{XPR}(1)=A M Z$ & Mass of the $Z$. \\
\hline $\mathrm{XPR}(2)=\mathrm{AMH}$ & Mass of the Higgs boson. (dummy for KEYGSW $=1,2$ ), \\
\hline $\mathrm{XPR}(3)=\operatorname{AMTOP}$ & Mass of the top quark. (dummy for KEYGSW $=1,2$ ), \\
\hline $\mathrm{XPR}(4)=\mathrm{GV}$ & Vector coupling constant $g_{V}$ of the $\tau$ to the $W$ in the $\tau$ decay. \\
\hline $\mathrm{XPR}(5)=\mathrm{VA}$ & $\begin{array}{l}\text { Axial coupling constant } g_{A} \text { of the } \tau \text { to the } W \text { in the } \tau \text { decay. } \\
\text { Note that } g_{V}=1 \text { and } g_{A}=-1 \text { represent the standard } V-A \\
\text { assignment. }\end{array}$ \\
\hline $\operatorname{XPR}(6)=\operatorname{SWSQ}$ & $\begin{array}{l}\text { Coupling constatnt } \sin ^{2} \theta_{W}(\text { dummy for } \mathrm{KEYGSW}=4) \text {. One } \\
\text { has to assign SWSQ and GAMMZ for KEYGSW }=0,1 .\end{array}$ \\
\hline $\mathrm{XPR}(7)=$ GAMMZ & Width of the $Z$ (dummy for KEYGSW $=4$ ). \\
\hline $\mathrm{XPR}(8)=\mathrm{AMNUTA}$ & $\begin{array}{l}\text { Mass of the } \nu_{\tau} \text {, used in } \tau \text { decay. It cannot be set exactly at zero } \\
\text { because of numerical stability (massive kinematics is used). }\end{array}$ \\
\hline $\operatorname{XPR}(9)=$ AMNEUT & $\begin{array}{l}\text { Mass of the } \nu \text { 's in the } \nu \text { generation mode, or the final state quark } \\
\text { mass in the quark mode. Because of the massive kinematics used } \\
\text { in the program it cannot be set exactly at zero. }\end{array}$ \\
\hline $\mathrm{XPR}(11)=\mathrm{XKO}$ & $\begin{array}{l}\text { Soft/Hard photon cut-off (in beam energy units) for the single } \\
\text { bremsstrahlung modes; otherwise dummy. }\end{array}$ \\
\hline $\mathrm{XPR}(12)=\mathrm{VVMIN}$ & $\begin{array}{l}\text { Minimal energy of the photon (in beam energy units) to be gener- } \\
\text { ated in multiphoton generation; typically below } 10^{-5} \text {. It should } \\
\text { be at least a factor of } 10 \text { smaller than the detector energy de- } \\
\text { tectability threshold. }\end{array}$ \\
\hline $\mathrm{XPR}(13)=\mathrm{VVMAX}$ & $\begin{array}{l}\text { Limit on the initial state photons phase space. VVMAX }=1 \\
\text { whole phase space. See also Refs. }[6,8] .\end{array}$ \\
\hline $\mathrm{XPR}(14)=\mathrm{XKODEC}$ & $\begin{array}{l}\text { Soft photon energy cut-off for the bremsstrahlung in the } \tau \text { decay. } \\
\text { See Ref. [11] for exact definition. }\end{array}$ \\
\hline
\end{tabular}




\section{Generation/Production Mode}

In this mode, all parameters of KORALZ, except for MODE $=0$, are ignored. The produced Monte Carlo event is stored in the common block

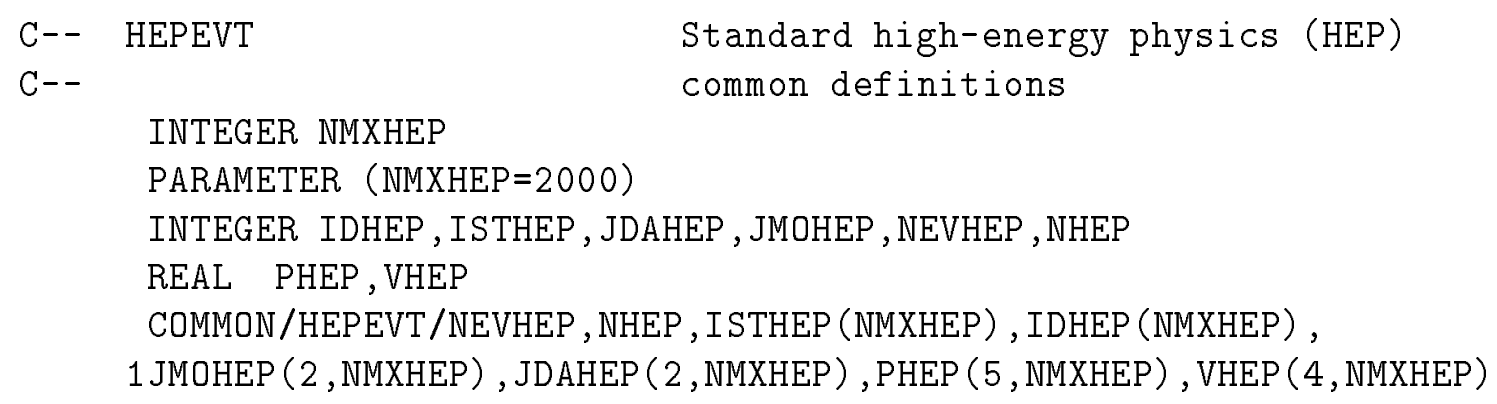

The common block HEPEVT contains a complete set of all initial and final state flavours and four-momenta encoded in the standard conventions [5]. It contains information on the $e^{ \pm}$beams, on the $\tau^{+}, \tau^{-}, \gamma$ and finally on all the $\tau^{ \pm}$decay products including neutrinos. Conservation of total energy and momentum is explicitly obeyed. The intermediate particles like $W^{ \pm}$and $\rho^{ \pm}$can be kept in the record. If the user wants the $\pi^{0}$ to decay, it can be done with eg. the help of JETSET [24] program (version 7.2 or higher). In KORALZ itself, however, there is no explicit call to any JETSET subprogram and KORALZ can be run without loading the JETSET library.

In addition, the final state momenta for the production process alone, may be found in COMMON / UTIL4 / QP(4), QM(4), PH(4) or in, COMMON / MOMSE4 / AQF1(4), AQF2 (4), $\operatorname{ASPHUM}(4), \operatorname{ASPHOT}(100,4)$, NPHOTA where REAL $* 4$ QP , QM (AQF1, AQF2) are four-momenta of $\tau^{+}$and $\tau^{-}$and REAL $* 4$ PH (ASPHUM) is the momentum of the bremsstrahlung photon in the case of single bremsstrahlung or the sum of all photon momenta (in $G e V$ units). NPHOTA denotes the number of the generated photons. REAL $* 4$ matrix ASPHOT stores the four momenta of the photons. 2.helicities (chiralities) of $\tau^{+}$and $\tau^{-}$are stored in the COMMON / TAUHEL / HELT1, HELT2 as REAL*8 variables.

\section{Postgeneration Mode}

A call on KORALZ in this mode (MODE=1) may be optionally used in order to obtain information on the value of the integrated cross section and the list of the partial $\tau$ decay widths. They are obtained with a finite statistical error depending on the number of generated M.C. events and they correspond strictly to the production and decay matrix elements used in the actual M.C. generation ${ }^{3}$. All of this information is printed in the output file and it is also provided to the user through parameter matrices XPR and NPR, see table below.

\footnotetext{
${ }^{3}$ For generation, however, different values of the branching ratios given in the COMMON /TAUBRA/ and /TAUNPI/ (see subroutine INITDK) are used. The values given here are not determining the proportion of the different channels in the decay sample but rather are used as (dummy) input parameters to improve efficiency of the M.C. algorithm in the decay routines.
} 


\begin{tabular}{|c|c|}
\hline Parameter & Meaning \\
\hline $\operatorname{NPR}(10)$ & Number of generated $\tau$ pairs. \\
\hline $\operatorname{XPR}(10)$ & Total cross section in $\mathrm{cm}^{2}$. \\
\hline $\mathrm{XPR}(16)$ & Total cross section in $\mathrm{cm}^{2}$ (the same as XPR(10)). \\
\hline $\mathrm{XPR}(17)$ & Total cross section in $n b$. \\
\hline $\mathrm{XPR}(18)$ & Statistical error on the total cross section in $n b$ units. \\
\hline $\mathrm{XPR}(21)$ & Partial width of $\tau \rightarrow \nu e \bar{\nu}$ (in lowest order $\Gamma(\tau \rightarrow \nu e \bar{\nu})$ units). \\
\hline $\operatorname{XPR}(22)$ & Partial width of $\tau \rightarrow \nu \mu \bar{\nu}$ (in lowest order $\Gamma(\tau \rightarrow \nu e \bar{\nu})$ units). \\
\hline $\mathrm{XPR}(23, \ldots)$ & $\begin{array}{l}\text { Partial widths of further } \tau \text { decay modes accordingly to list given } \\
\text { in Ref. [11]. }\end{array}$ \\
\hline
\end{tabular}

\section{Possible Future Developments}

As is clear from the list of the limitations given in the Introduction, a number of developments are still possible:

1. Complete exponentiation of all QED bremsstrahlung including interference.

2. Complete $\mathrm{O}(\alpha)$ radiative corrections in the semi-leptonic $\tau$ decays, or proof that presently available corrections are precise enough.

3. Inclusion of still missing $\tau$ decay modes, in particular $\tau^{ \pm} \rightarrow K^{ \pm} K^{0} \nu$ and $\tau \rightarrow 5 \pi \nu$ including effects of omega.

4. Implementation of transverse spin polarisation effects.

5. Tests comparing various (approximate and exact) spin implementations [17]. 


\section{Acknowledgements}

One of the authors (Z.W.) acknowledges a warm hospitality of the Centre de Physique des Particules de Marseille where he was able to assemble and test the first version of the program, the Max Planck Institute Munich and CERN-TH Geneva, where the program was later developed. Authors of the program are indebted to E. Barberio, F. Boillot, G. Bonneaud, D. DeLaney, H. Burkhardt, P. Colas, R. Decker, M. Gruenewald, M. Jeżabek, J.H. Kühn, A.M. Lutz, L. Mirabito, R. Miquel, E. Mirkes, K. Mönig, W. Płaczek, E. Richter-Wąs, C. Shio, G. Siopsis, M. Skrzypek, U. Stiegler, B. van Eijk, B. Wysłouch and S. Yost for useful discussions, for the help in organising the program, in testing/debugging the program, and finally in extending the program to neutrino and quark modes and some $\tau$ decay modes. Work supported in part by the Polish Government Grants KBN203809101 and KBN-223729102. Work supported in part by the United States DOE contracts DE-FG05-91ER40627 and DE-AC03-76SF00515.

The authors have benefited from interactions with the SLC/LEP Physics Working Groups and they thank Profs. J. Ellis, G. Altarelli, J. Dorfan, G. Feldman and F. Dydak for giving them the opportunity to realize these interactions. The authors are grateful to Profs. D. Bardin, W. Hollik, B. Kniehl, T. Riemann, M. Sachwitz and R. Stuart for assistance in interfacing their respective electroweak corrections libraries to KORALZ.

\section{Appendix A}

\section{Interface of Electroweak Libraries to KORALZ}

The interface between part (1) and part (2) or (3), see Section 2, of KORALZ has to be clearly defined. Physics of weak interactions and many details of $\tau$ decays will be probed experimentally with the higher precision, and thus it may be necessary to change or replace parts (2) or (3). The interface between the $\tau$ decay library TAUOLA and KORALZ is explained in detail in Ref. [11]. Here, let us concentrate on the interface between KORALZ and electroweak libraries.

In principle, the QED corrections are part of the electroweak corrections and, as such, have to be calculated in the renormalizable quantum field theory of Glashow-WeinbergSalam. In fact, such a separation can be done only up to the $\mathrm{O}(\alpha)$. At higher orders, it can be done in next-to-leading-log approximation. The pure QED non-logarithmic terms of $\mathrm{O}\left(\alpha^{2}\right)$ alone do not have any phenomenological value at LEP, unless they are combined with the terms of the same order coming from the complete electroweak theory. Fortunately, such terms are too small anyway, to be seen experimentally.

In addition, let us keep in mind, that one of the goals of the LEP experiments is to check the Standard Model. Consequently, the electroweak non-QED corrections are the real target of the measurements.

One of the convenient ways of dealing with the electroweak corrections is the following [19]:

1. Construct the Monte Carlo program for QED, including only this part of electroweak corrections which can be absorbed into redefinition of the coupling constants and $Z$ width (crude differential cross section). 
2. Provide an electroweak library which calculates all electroweak corrections, but excluding QED bremsstrahlung. Define routine calculating the two-body differential cross section $d \sigma / d \Omega(s, \Theta)$ with all non-QED corrections.

3. Design an algorithm (reduction procedure) which combines photon momenta with incoming and outgoing fermion momenta in such a way that the effective two-body hard scattering process is defined [19].

In the KORALZ, we follow the outline described above, and the electroweak corrections are implemented by means of rejection. For every event, we first generate a M.C. event assuming a simplified form of the (crude) cross section; that is, we include only those electroweak corrections which can be absorbed into energy independent coupling constants and $Z$ width.

In the second step, by means of rejection, we introduce the remaining and numerically small (or an overall normalisation) part of the electroweak corrections. To this end, we calculate for each event a weight (with the help of hard scattering process variables) equal to the ratio of the complete electroweak cross section calculated in a particular point of the phase space and the previously used crude one. In principle, there is no restriction on the crude cross section; good approximation improves only the efficiency of the algorithm. However, the angular distribution of the crude cross section due to technical reasons must have always the following form: $1+\cos ^{2} \theta+$ Const $_{1} \cdot \cos \theta+$ Const $_{2} \cdot \sin ^{2} \theta$. More precisely, such an algorithm can be used for implementation of relatively small corrections e.g., of the $\mathrm{O}(\alpha)$. The absolute error in their implementation, due to approximations used in reduction procedure, is of the $\mathrm{O}\left(\alpha^{2}\right)$. The overall normalisation is an exception and there is no error in its implementation by the method described above.

To interface any electroweak library to KORALZ the following routines will have to be replaced with the new ones:

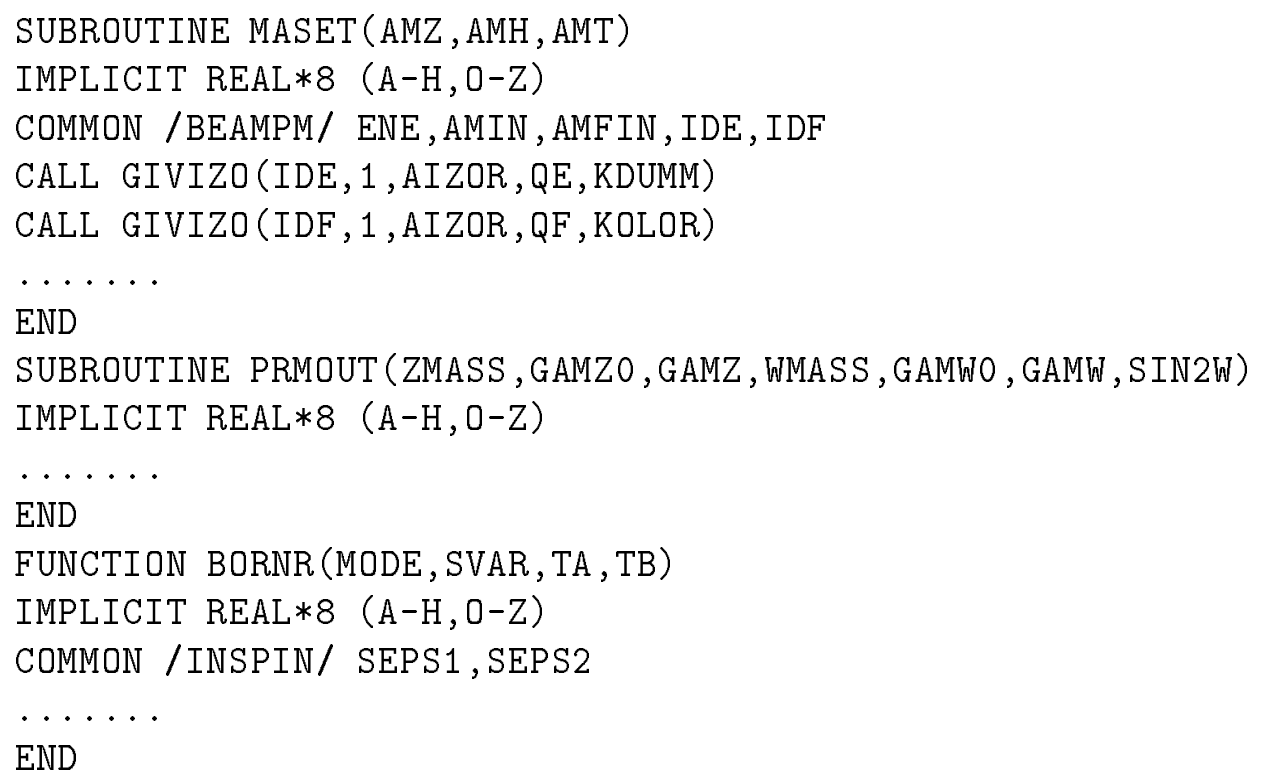


Calls to all those routines are already defined within the KORALZ library and the only communication between KORALZ and electroweak libraries is the following: SUBROUTINE MASET initialize electroweak library. Variables AMZ, AMH, AMT, denote the values (in GeV) of $Z$, Higgs and top masses which are transmitted from KORALZ to the electroweak library, some extra initialization parameters of the electroweak libraries are set and printed by this call as well. Also, the content of the common /BEAMPM/ is predefined in KORALZ before any call on the electroweak library. The variables ENE, AMIN , AMFIN, IDE, IDF denote beam energy, electron and final state masses (all in $\mathrm{GeV}$ ), and flavour identifiers for the beams. Final state fermions IDF (IDE) $=1,2,3,4$ denote neutrino, lepton, up-type quark and down-type quark. The routine GIVIZO calculates from the flavour identifiers IDE, IDF charges QE, QF and color factor KOLOR. All these parameters may be needed to initialize the electroweak library.

KORALZ calls on routine PRMOUT (ZMASS , GAMZO, GAMZ, WMASS , GAMW0, GAMW , SIN2W), of the electroweak part which transmits some parameters of electroweak corrections wherever they may turn out to be needed. The (output) parameters of routine PRMOUT denote respectively: $Z$ mass, lowest order and corrected $Z$ width, $W$ mass, lowest order and corrected $W$ width, and finally $\sin ^{2} \theta_{W}$.

Functionally, the most important part of the interface is the function BORNR (MODE, SVAR, COSTHE, TA, TB) which calculates two-body cross section. We define our function BORNR using conventions of spin quantization as in Ref. [25], including final mass terms. The meaning of its parameters is the following: MODE denote the mode of the operation. For $\mathrm{MODE}=0$, a crude distribution should be calculated, otherwise complete electroweak corrections should be included. The $S V A R=s$ denotes a Mandelstam variable of the reduced process, $\mathrm{COSTHE}=\cos \theta$, an angle between directions of $\tau^{+}$and $e^{+}$. Variables TA, TB denote polarization states of the final state fermions. They denote respectively twice helicity (chirality) of $\tau^{+}$and minus twice helicity of $\tau^{-}$(in general first and second final state fermions). In KORALZ, only values $\mathrm{TA}, \mathrm{TB}= \pm 1,0$ are used. According to the same conventions, spin polarization states SEPS1, SEPS2 of the beams are defined. In this case, any value between -1 and +1 can be provided from KORALZ.

Let us note that if it will be be necessary to change/add an electroweak library in the future, the overall normalisation of the function BORNR has to be chosen in such a way that for the unpolarized muon cross section (in the massless limit), and pure s-channel $\gamma$, the cross section is given by the following formula $1+\cos ^{2} \theta$. Finally, for the sake of tests and backward compatibility, the interface to the electroweak library should be organized in such a way that, once input of KORALZ KEYGSW is chosen to be 1 (no electroweak corrections), the interface returns exactly the same distribution as the one presently available in KORALZ. For this purpose it is compulsory that additional input parameters are transmitted from KORALZ to the electroweak library interface. Consequently, the common blocks /GSWPRM/ SWSQ , AMW, AMZ, AMH, AMTOP, GAMMZ and /KEYSET/ KEYGSW, KEYRAD , KEYTAB should be used in routine BORNR. Variable SWSQ denotes $\sin ^{2} \theta_{W}$, and AMZ, GAMMZ denotes $Z$ mass and width. $Z$ coupling constants are calculated from $\sin ^{2} \theta_{W}$ following conventions defined in [10]. In this last case, the call on the routine MASET initializing the electroweak library is not executed. 
At present, KORALZ is interfaced with the three electroweak libraries; the DubnaZeuthen group [12], see also [23], B. Kniehl and R. Stuart [13] and W. Hollik. The third code by W. Hollik is unpublished and corresponds to Ref. [15].

\section{Appendix B}

\section{Extension of the Program to the $q$ and $\nu$ Cases $^{4}$}

In order to switch from the $e^{+} e^{-} \rightarrow \mu^{+} \mu^{-}$process to $e^{+} e^{-} \rightarrow \nu \bar{\nu}$, we change the final state couplings (i.e., reverse the weak isospin, set the charge to 0 ) and add the $W$ exchange in the $t$-channel graph to the $Z$ exchange in the $s$-channel graph.

Because the $W$ exchange contribution is small, it is enough to use the following leading logarithmic approximation. We neglect emission of the photons from the $W$ and assume the $t$-channel $\gamma-W$ boxes can be described by the same function as the $Z-e-e$ vertex corrections. The first approximation has been proved to be very good [26]. In the crude cross section, we use the contact approximation for the $W$ exchange. The remaining very small correction to the contact approximation is introduced later by rejection. See Refs. $[27,28]$ for more details.

For the quarks in the final state, we proceed as follows: We change accordingly the charge and the isospin of the final state fermions and add the color factor. In routine BORNR, we add also an overall factor representing final state QCD corrections for the total cross section eg, $1+\alpha_{S}(s) / \pi+\alpha_{s}^{2}(s)\left(1.98-N_{F} \cdot 0.115\right) ;\left(s=4 \mathrm{~PB} 1(4)^{2}\right)$ in order to obtain proper normalisation of the cross section. ${ }^{5}$ The specific top mass dependent final state weak vertex corrections are switched on in the $b$ quark case. In the case of quarks, final state bremsstrahlung in our program can be generated at the free quark level only. This is known (see eg. [30]) to be a rather poor approximation, typically overestimating QED bremsstrahlung by a factor of two due to lack of gluonic corrections.

\section{Appendix C}

\section{The Algorithm Saving CPU Time}

A definite drawback of the Monte Carlo programs generating series of events for the physical processes is the limited possibility of playing with input parameters and looking into their influence on the quantities directly related with the measurements in a given experiment. It is particularly true for full scale detector simulation when the computer time needed by Long Chain (LC) ${ }^{6}$ to process one event is sometimes more than one

\footnotetext{
${ }^{4}$ The extension of KORALZ to the $\nu$ case was developed with the active collaboration from Laurent Mirabito and Paul Colas.

${ }^{5}$ Note that this overall scaling factor does not take into account terms which distinguish between QCD corrections to axial and vector couplings of the $Z$ [29]. In the case of Z0POLE library, we use the function ALPHAS from the program of Ref. [13], in the case of the DIZET library, we use an overall QCD correction factor [12]; see also demonstration output for the numerical value.

${ }^{6}$ This name is used to describe the procedure of: (1) digitalization, i.e., complete simulation of the detector response to the particular event (to get an event in a form identical to the one registered in the experiment); (2) evaluation back of the physical content of the event by means of the reconstruction programs, i.e., procedures prepared for the reconstruction of the events registered in the experiment.
} 
minute of the typical computer CPU time. Thus, physical events are generated only once, then they are processed through the Long Chain and are archived on the tape for any further usage.

This leaves very little room, if any, for the analysis of the influence of variation of the input parameters (such as Higgs or top masses or addition of another heavy fermion family or second heavy boson) on the generated sample of events. Let us sketch a possible solution of the problem, using as an example the process $e^{+} e^{-} \rightarrow \tau^{+} \tau^{-}$.

The differential cross section after inclusion of the radiative corrections differs significantly from the Born cross section. Completely new final states, such as $\tau^{+} \tau^{-} \gamma$, which cannot be obtained by any means from (LC) events, generated using the Monte Carlo program for the cross section in Born approximation. Fortunately at LEP energies, appearance of the extra particles (in practice, only photons) in the final state is caused by the best known (to some extent non-interesting) and well-established part of the Standard Model, i.e., the QED.

On the contrary, the effects of the modifications in the non-QED part of the Standard Model can be represented as

$$
\frac{d \sigma^{\text {modif }}}{d \Omega}=\frac{d \sigma^{O R I G I N A L}}{d \Omega} w^{\text {modif }}(\Omega)
$$

with a positive function $w^{\text {modif }}(\Omega)$ which is close to 1 essentially ${ }^{7}$ all over the phase space.

This opens a possible way of dealing with the modification in the Standard Model in a way which does not require repetition of the Long Chain analysis.

The complete scenario can be viewed as follows:

1. The series of events are generated according to a certain configuration of parameters in the Physics Monte Carlo event generator.

2. The Long Chain analysis is performed on those events.

3. All events which passed the Long Chain (LC events) are archived on the tape.

4. A new set of input parameters (or modified version of the electroweak model) is chosen.

5. For every LC event, at the corresponding point in the phase, space we recalculate the value of the differential cross section which was used in the original generation. At the same point, we calculate a new value of the differential cross section corresponding to modified parameters. We assign the ratio of these two cross sections, $w^{\text {modif }}$, to every LC event. With this weight we treat the entire series of old LC events as if it was generated with the new parameters.

\footnotetext{
${ }^{7}$ This statement is not valid only in the small regions $\left(\cos \theta= \pm 1\right.$ and $\left.\sqrt{s} \simeq M_{Z} \mp 15 G e V\right)$ of the phase space where the differential cross section approaches zero. Change in the input parameters may shift position of this zero and there the function $w^{\text {modif }}(\Omega)$ may differ significantly from 1 . These regions (of cross section very close to 0 ) give a negligible contribution to observables and may be excluded from this consideration.
} 
Applicability of such a scenario requires some precautions and prearrangements. From the user side, the stored LC event has to include enough information to recalculate the value of the differential cross section at the corresponding point of the phase space.

On the other hand, in the Physics Monte Carlo program, there should exist a clearly defined function, easy for further modifications and extensions, which calculates the (QED reduced) differential cross section out of momenta of final state particles.

In the case of KORALZ, the function BORNS (MODE, SVAR, COSTHE, T1, T2) of the same parameters as function BORNR described in Appendix A may be used for this purpose. For every generated event, parameters of the function BORNS have to be stored. The numerical value of T1, T2 may be picked up from the common block TAUHEL, T1=HELT1, T2=-HELT2. The SVAR, COSTHE can be obtained by the additional call to routine BDRESS (1, PPBT , PMBT , SVAR, COSTHE) after an event is generated. The PPBT (4), PMBT (4) are declared as $R E A L * 8$. Then, for each archived $\mathrm{LC}$ event, the weight $w^{\text {modif }}$ can be calculated using the BORNS function and initializing KORALZ once with the old and once with the new set of the input parameters. The function BORNS calculates the differential distribution of the final state fermions without QED bremsstrahlung corrections but including weak corrections.

In an analogous way, although a bit more involved the nontrivial transverse spin correlations between decay products of $\tau^{+}$and $\tau^{-}$could be included into series of LCA events. However, at present, we recommend to use for this purpose the KORALB [22] generator at Born level.

\section{Appendix D}

\section{Numerical Tests of the Program}

A program of this size and complexity, as is the case with KORALZ, requires exhaustive testing before it can be considered reliable. So far the following tests and groups of tests were reproduced or performed anew.

1. In Ref. [11], tests on the $\tau$ decays and, in Ref. [9], tests of approximate algorithm for radiative corrections in decays are reported.

2. In the description of the YFS2/YFS3 [6] generator for the initial state multiphoton bremsstrahlung, some tests are reported. Some further tests are forthcoming [31].

3. Comparison of results by KORALZ with another $\nu$ generator can be found in Refs. $[27,28]$.

4. The prediction of KORALZ for the forward-backward asymmetry of muons is compared with the analytical calculations in Ref. [32], see also [21].

5. The $\tau$ polarization observables are compared with semi analytical calculations, including also the leading logarithmic bremsstrahlung corrections in decay, in Ref. $[33]$.

6. A multitude of other comparisons with the semi analytical program CALASY [34], in particular for the total cross section, can be found also in [35]. 
7. The further options of calculations of electroweak corrections are compared [23, 21] with the ones actually used in KORALZ.

8. The exhausting tests on comparison of spin implementation in KORALZ and complete density matrix treatment as in KORALB [17] are not yet completed.

We encourage the user to reproduce, at least, those of these tests which are most relevant for her or his applications. Note that we are unable to quote a single number for precision of our program. Due to the general principles, it can be given only for well defined observables and experimental cuts [36].

\section{References}

[1] G. Altarelli et al., "Precision Tests of the Electroweak Theory at the $Z^{0}$ ", Physics at LEP, ed. J. Ellis and R. Peccei, Vol. 1, page 167, CERN, Geneva 1986.

[2] Proceedings of the SLC workshop on experimental use of the SLAC Linear Collider, SLAC-Report-247, (1982).

[3] S. Glashow, Nucl. Phys. 22 (1961) 579; A. Salam, Elementary Particle Theory, ed. H. Svartholm (Almquist, Stockholm, 1968); S. Weinberg, Phys. Rev. Lett. 19 (1967) 1264.

[4] S. Jadach, B.F.L. Ward, and Z. Wạs, Comp. Phys. Commun. 66 (1991) 276.

[5] M. Aguilar-Benitez et al. (Particle Data Group), "Review of Particle Properties", Phys. Lett. B239 (1990) 1.

[6] S. Jadach and B.F.L. Ward, Comp. Phys. Comm. 56 (1990) 351.

[7] S. Jadach and B.F.L. Ward, Phys. Lett. B274 (1992) 470.

[8] S. Jadach, B.F.L. Ward and Z. Wạs, "YFS3 Monte Carlo", available from authors, to be published in Comp. Phys. Commun.

[9] E. Barberio, B. van Eijk and Z. Wạs, Comp. Phys. Comm. 66 (1991) 115; E. Barberio, and Z. Wass, "PHOTOS-A Universal Monte Carlo for QED Radiative Corrections: Version 2.0", CERN preprint (1993), CERN TH-7033/93.

[10] F.A. Berends, R. Kleiss and S. Jadach, Comp. Phys. Commun. 29 (1983) 185.

[11] S. Jadach, J.H. Kühn and Z. Wạs, Comp. Phys. Commun. 64(1991) 275; S. Jadach, M. Jeżabek, J.H. Kühn and Z. Wạs, Comp. Phys. Commun. 70(1992) 69; R. Decker, S. Jadach, J.H. Kühn and Z. Wạs, Comp. Phys. Commun. 76(1993) 361.

[12] D.Y. Bardin et al., Comp. Phys. Comm. 59 (1990) 303. See also D.Y. Bardin et. al. "ZFITTER - An Analytical Program for Fermion Pair Production in $e^{+} e^{-}$Annihilation", CERN preprint CERN-TH 6443/92 (1992). 
[13] B. Kniehl, R. Stuart, Comp. Phys. Commun. 72 (1992) 175.

[14] O. Adriani et. al., Phys. Lett. B295 (1992) 337.

[15] W. Hollik, Fortschr. Physik 38 (1990) 165; W. Hollik, "Renormalization of the Standard Model" MPI-Munich preprint MPI-PH-93-21, to appear in Precision tests of the Standard Model, advanced series on directions in high-energy physics, ed. by Paul Langacker, World Scientific; W. Hollik, "Predictions for $e^{+} e^{-}$processes" MPIMunich preprint MPI-PH-93-21, to appear in Precision tests of the Standard Model, advanced series on directions in high-energy physics, ed. by Paul Langacker, World Scientific.

[16] W. Hollik, M. Martinez, in preparation.

[17] S. Jadach, Z. Wạs in preparation.

[18] Z. Wa̧s, Acta Phys. Polon. B18 (1987) 1099.

[19] F. Boillot and Z. Wạs, Z. Phys C43 (1989) 109.

[20] S. Jadach and Z. Wạs, Phys Lett. 219 (1989) 103; J.H. Kühn, S. Jadach, R.G. Stuart and Z. Wạs, Z. Phys. C38 (1988) 609; J.H. Kühn and R. G. Stuart, Phys. Lett. B200 (1988) 360.

[21] S. Jadach, B.F.L. Ward and Z. Wạs, Phys. Lett. B257 (1991) 213.

[22] S. Jadach and Z. Wạs, Comp. Phys. Commun. 36 (1985) 191; S. Jadach, Z. Wạs, ibid. 64 (1991) 267 and CERN preprint (1994), in preparation.

[23] T. Riemann, M. Sachwitz and Z. Wạs, "A Comment on $\mathrm{O}\left(\alpha \alpha_{s}\right)$ Corrections and Phenomenology of LEP Experiments", CERN preprint (1990), CERN TH-5964.

[24] T. Sjöstrand, Comp. Phys. Commun. 27 (1982) 243, and Comp. Phys. Commun. 28 (1983) 229.

[25] S. Jadach, Z. Wạs, Acta Phys. Polon. B15 (1984) 1151.

[26] F.A. Berends et al., Nucl. Phys. B301 (1988) 583.

[27] P. Colas, L. Mirabito and Z. Wạs, Munich preprint (1989) MPI PAE-Exp. El. 211.

[28] P. Colas, R. Miquel and Z. Wąs, Phys. Lett. B246 (1990) 541.

[29] J.H. Kühn et al., "Heavy Flavours", Z-PHYSICS at LEP 1 eds. G. Altarelli, R. Kleiss and C. Verzegnassi, Vol. 1, page 267, CERN, Geneva 1989.

[30] P. Mättig, H. Spiesberger, W. Zeurner, "On the Comparison of Matrix Element Calculations of $\mathrm{O}\left(\alpha \alpha_{s}\right)$ with the Measurement of Photon Emission in Hadronic $Z^{0}$ Decays", CERN preprint CERN-PPE/93-119 (1993). 
[31] S. Jadach et al., in preparation.

[32] S. Jadach and Z. Wạs, Phys. Rev. D41 (1990) 1425.

[33] S. Jadach et al., "The $\tau$ Polarization Measurement", Z-PHYSICS at LEP 1, eds. G. Altarelli, R. Kleiss and C. Verzegnassi, Vol. 1, page 235, CERN, Geneva 1989.

[34] S. Jadach and Z. Wạs, "CALASY Program", unpublished. The approach to QED was developed in Ref. [21]; a short description can be found in: Z-PHYSICS at LEP 1 eds. G. Altarelli, R. Kleiss and C. Verzegnassi, Vol. 3, page 58, CERN, Geneva 1989 .

[35] R. Kleiss et al., "Monte Carlos for Electroweak Physics" in: Z-PHYSICS at LEP 1 eds G. Altarelli, R. Kleiss and C. Verzegnassi, Vol. 3, page 1, CERN, Geneva 1989.

[36] S. Jadach, Z Wạs, "Physics Monte Carlo for $\tau$ Lepton-Question of Theoretical and Technical Precision" Proceedings of Second Workshop on tau Lepton Physics' p. 37, ed. K.K. Gan, World Scientific 1992.

\section{TEST RUN OUTPUT}

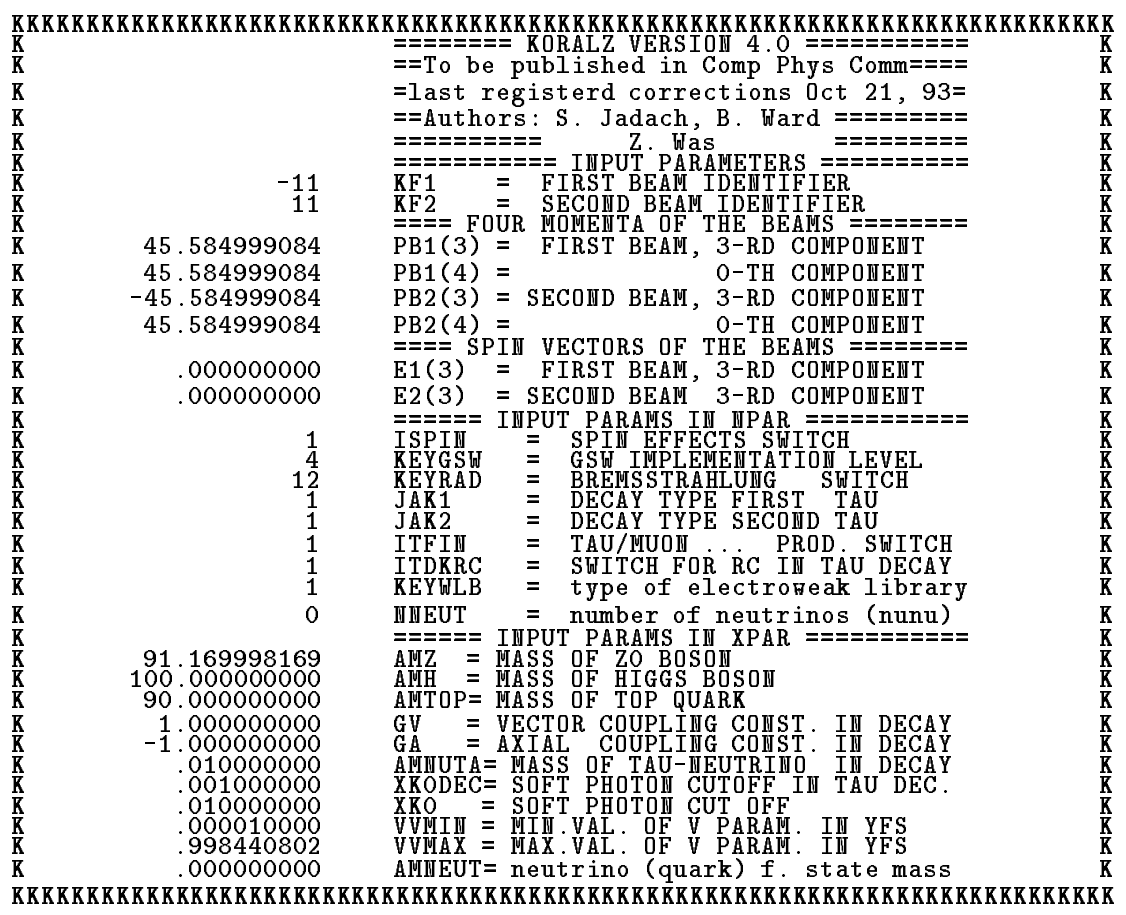




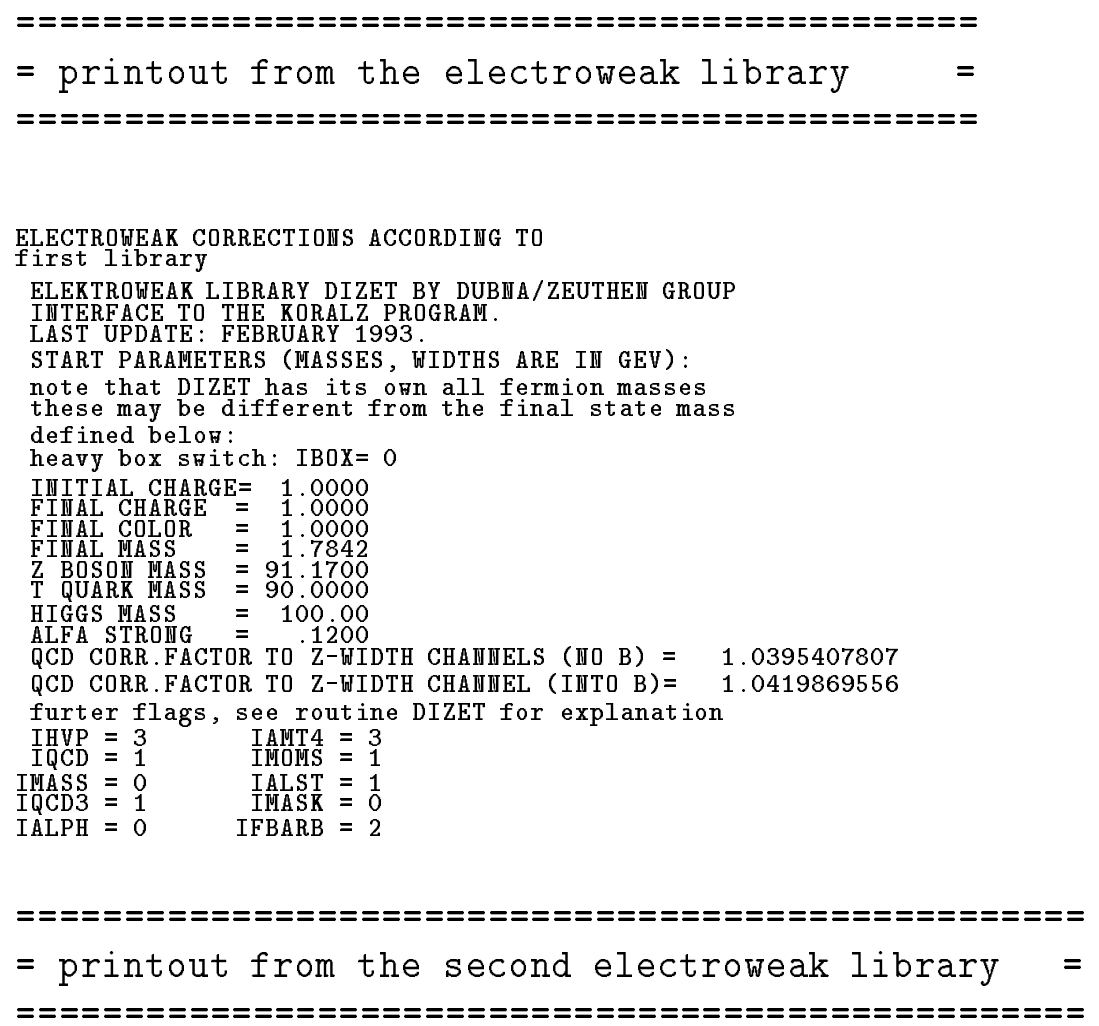

\begin{tabular}{|c|c|c|}
\hline **** & 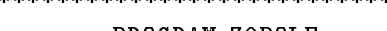 & $* * *$ \\
\hline$* * *$ & PROGRAM ZOPOLE & $* * *$ \\
\hline $\begin{array}{l}* * * \\
* * *\end{array}$ & ROBIN AUTHOR & $\begin{array}{l}* * * \\
* * *\end{array}$ \\
\hline$* * *$ & CERII & $* * *$ \\
\hline$* * *$ & QCD CORRECTIOIIS & $* * *$ \\
\hline$* * *$ & BERID A ${ }_{\text {DESY }}^{\text {KHIEHL }}$ & $\begin{array}{l}* * * \\
* * *\end{array}$ \\
\hline$* * *$ & & $* * *$ \\
\hline **** & LAST UPDATE : JUNE, 1992 & $* * *$ \\
\hline$* * * *$ & & $\begin{array}{l}* * * \\
* * *\end{array}$ \\
\hline$* * * *$ & $* * * * * * * * * * * *$ & ***** \\
\hline$* * * *$ & LAST UPDATE OF IHTERFACE: & $* * *$ \\
\hline$* * *$ & IIOVEWBER 2, 1993 & $* * *$ \\
\hline$* * *$ & & $* * *$ \\
\hline
\end{tabular}

IIIPUT PARAMETERS; OII-SHELL SCHEME ZO MASS $=91.1700$ $\begin{array}{ll}\text { HIGGS MASS } & 100.0000 \\ \text { TOP QUARK MASS } & 90.0000\end{array}$

IISCHEM = 2 HAS BEEN SELECTED; THE MS-BAR RENORMALIZATION SCHEME WILL BE USED.

TII THE MS-BAR SCHFHE T HOOFT MASS $=91.1700$

$\begin{array}{ccr}\text { III THE MS-BAR SCHEME THE REHORMALIZED PARAMETERS ARE. } & = \\ \text { 1/ALPHA } & = & 127.96342723 \\ \text { SII**2 W } & =2333169 \\ \text { W MASS } & = & 80.12902949 \\ \text { Z MASS } & = & 91.51370243\end{array}$

POSITION OF THE POLE ON THE COMPLEX S-PLANE;

$S$ POLE $=8305$

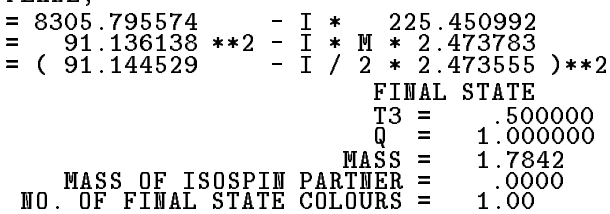

EXTERIAL FERMIOIS: IIITTAL STATE

$\mathrm{T} 3=$ Q 1.500000 


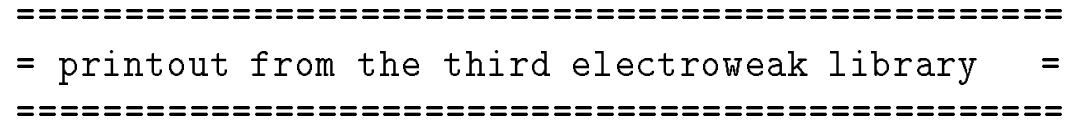

PHOTOS QED Corrections in Particle Decays

Monte Carlo Program - by E. Barberio, B. van Eijk and Z. Was From version 2.0 on - by E.B. and Z.W.

Internal input parameters:

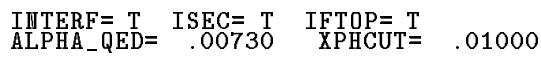

option rith interference is active option with double photons is active emision in $t$ tbar production is active

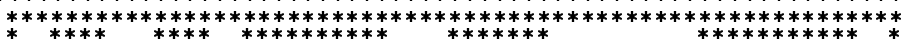

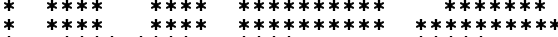

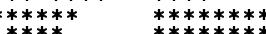

$* * * *$

$\underset{* * * *}{* * * * * * * *}$ $* * * * * *$

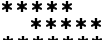

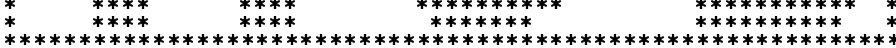

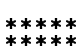

$* * * * * * * * * *$

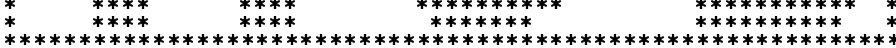

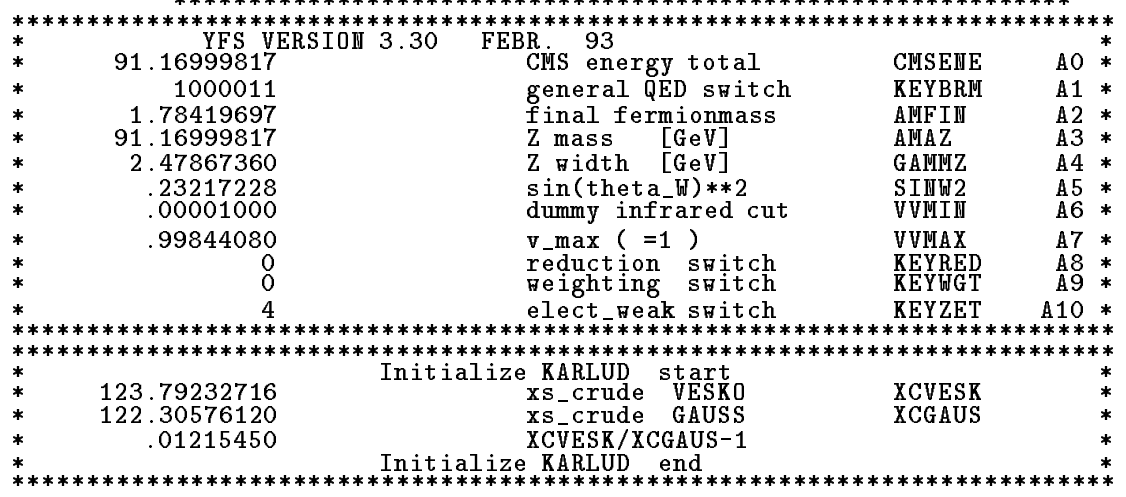




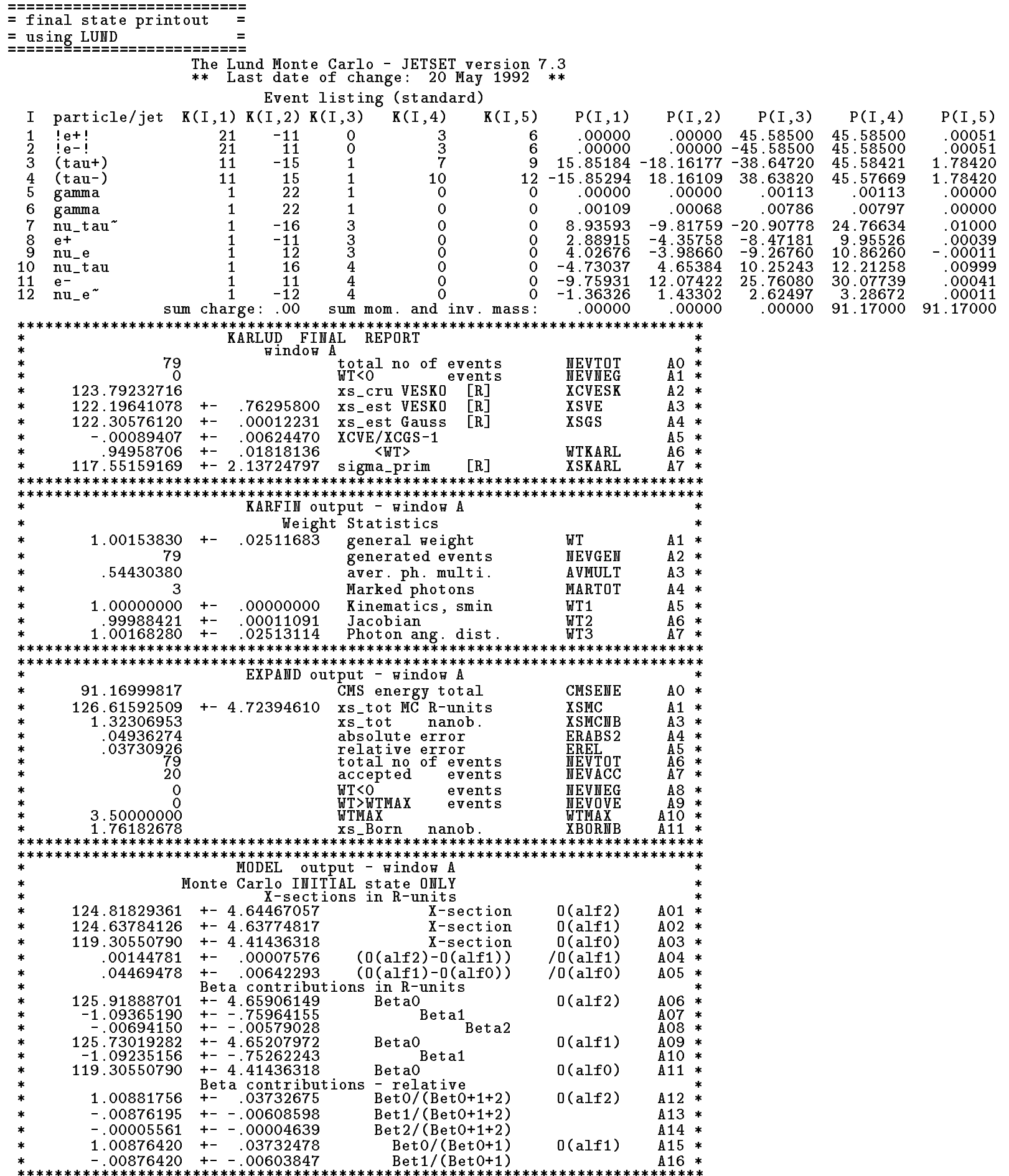




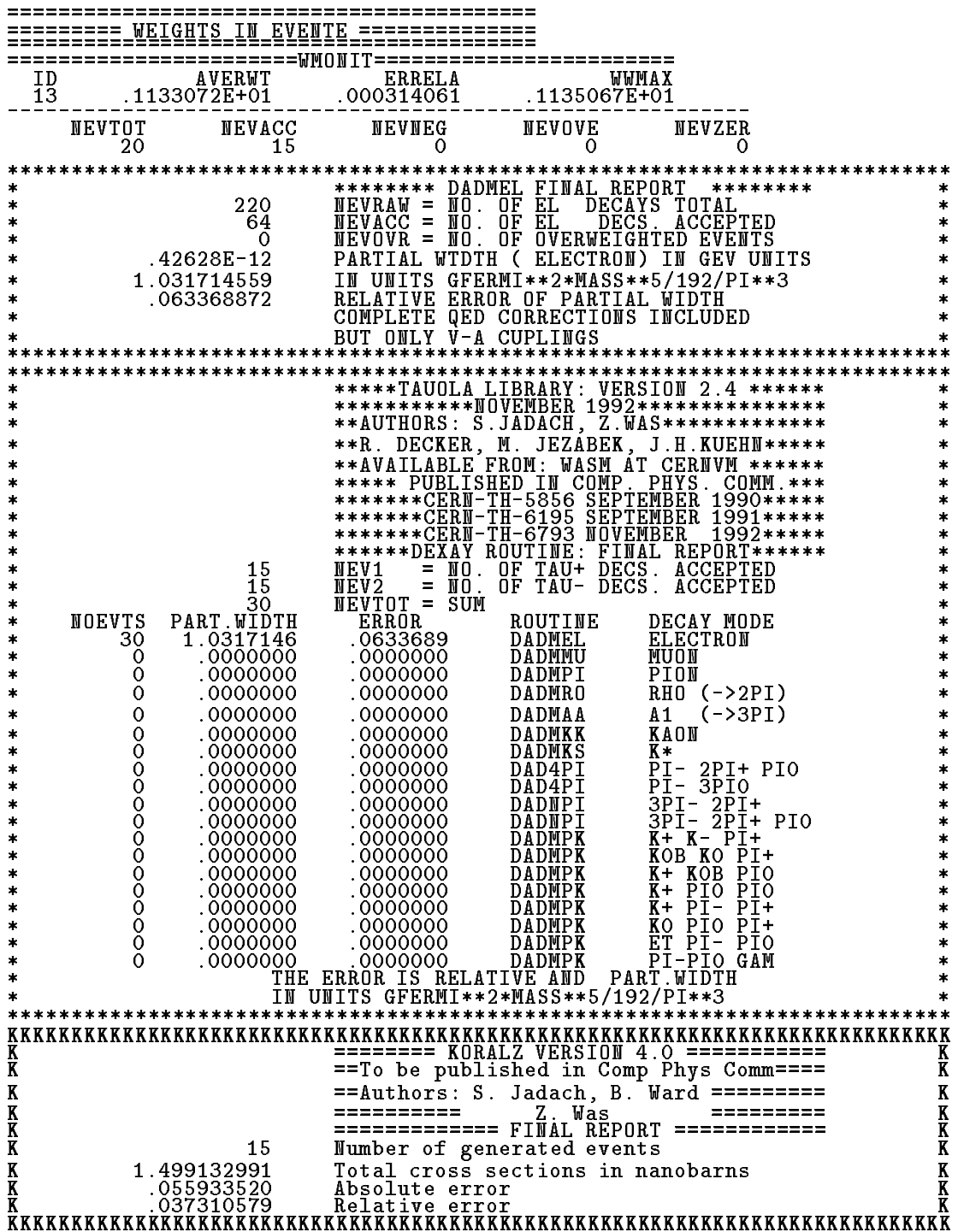

